\title{
Effects of deferoxamine on the osteogenic differentiation of human periodontal ligament cells
}

\author{
SEN MU $^{1}$, SHUANLONG GUO ${ }^{2}$, XIANG WANG ${ }^{3}$, YUANBO ZHAN $^{4}$, \\ $\mathrm{YING} \mathrm{LI}^{4,5}$, YING JIANG ${ }^{6}$, RUIMIN ZHANG ${ }^{1}$ and BIN ZHANG $^{4,5}$ \\ ${ }^{1}$ Department of Periodontology and Oral Mucosa, The Second Affiliated Hospital of Harbin Medical University, \\ Harbin, Heilongjiang 150086; ${ }^{2}$ Department of Stomatology, Fenyang Hospital, Fenyang, Shanxi 032200; \\ ${ }^{3}$ Department of General Dentistry, Yinzhou Stomatology Hospital, Ningbo, Zhejiang 315000; ${ }^{4}$ Institute of \\ Hard Tissue Development and Regeneration, The Second Affiliated Hospital of Harbin Medical University, \\ Harbin, Heilongjiang 150086; ${ }^{5}$ Heilongjiang Academy of Medical Sciences, Harbin, Heilongjiang 150001; \\ ${ }^{6}$ Pediatric Department of Stomatology, Yinzhou Stomatology Hospital, Ningbo, Zhejiang 315000, P.R. China
}

Received January 1, 2017; Accepted August 17, 2017

DOI: $10.3892 / \mathrm{mmr} .2017 .7810$

\begin{abstract}
Hypoxia regulates a number of cell biological processes, including cell survival, development and differentiation. Deferoxamine (DFO), an oral chelator for blood transfusion patients, has been demonstrated to induce hypoxia and is frequently used as a hypoxia-mimicking agent. The purpose of the present study was to investigate the influence of DFO on the proliferation, migration and osteogenic differentiation of human periodontal ligament cells (hPDLCs). The effects of DFO on hPDLC viability and migration were measured using an MTT and wound healing assay. To characterize the hypoxia microenvironment, the expression of hypoxia-inducible factor- $1 \alpha$ (HIF-1 $\alpha)$ in hPDLCs treated with DFO was quantified using the reverse transcription-quantitative polymerase chain reaction (RT-qPCR). Subsequently, the osteogenic differentiation potential of DFO was determined by RT-qPCR of the mRNA of osteogenic markers (runt-related transcription factor 2 [Runx-2], osteopontin [OPN] and collagen type I [Col-1]). The alkaline phosphatase activity and mineral deposition were analyzed using alizarin red $\mathrm{S}$ staining. The MTT and wound healing assays demonstrated that low-concentrations of DFO had little impact on hPDLC viability and migration $48 \mathrm{~h}$ into the treatment. DFO upregulated the expression of hPDLC genes specific for osteogenic differentiation: HIF-1 $\alpha$, Runx-2, OPN and Col-1. Furthermore, formation of mineralized nodules was enhanced by DFO. The present study suggests that DFO provided favorable culture
\end{abstract}

Correspondence to: Dr Bin Zhang, Institute of Hard Tissue Development and Regeneration, The Second Affiliated Hospital of Harbin Medical University, 246 Xue Fu Road, Harbin, Heilongjiang 150086, P.R. China

E-mail: zbinyzz@163.com

Key words: deferoxamine, human periodontal ligament cells, hypoxia-inducible factor- $1 \alpha$, osteogenic differentiation conditions to promote the osteogenic differentiation and mineralization of hPDLCs. The mechanism underlying these alterations remains to be elucidated.

\section{Introduction}

Periodontal ligament (PDL) tissue, which originates from cranial neural-crest-derived ectomesenchymal cells (1), is a specialized fibrous connective tissue located between the cementum of the tooth root and the alveolar bone (2). PDL serves a role in anchoring teeth and maintaining the structural integrity of the periodontium (2). Human PDL cells (hPDLCs) form a heterogeneous mixture of cell types, including fibroblasts, cementoblasts, osteoblasts, epithelial cells, vascular endothelial cells, smooth muscle cells (SMCs) and certain types of neural cells (3). The presence of multiple cell types has led to a hypothesis that the PDL tissue may also contain stem cells that maintain PDL homoeostasis and regenerate adjacent periodontal tissues. It has been confirmed that multipotent stem cells of hPDLCs have the ability to differentiate into osteoblastic/cementoblastic cells and generate cementum/PDL-like tissue in vivo (4). Therefore, the application of hPDLCs may be a promising therapeutic method for the reconstruction of tissues damaged by periodontal diseases.

Hypoxia is commonly associated with pathologies including tissue ischemia, inflammation and solid tumors (5-7). Additionally, hypoxic microenvironments form in embryos, and adults and often create specific niches which could regulate cellular differentiation and multi-gene expression in verious cell types (8). Furthermore, hypoxia has been found to be a potent inducer of the expression of osteogenesis/mineralization genes (9). Cells have the ability to modulate their processes to adapt to specific hypoxic niches during development and tissue maintenance or repair (8).

Cells respond to hypoxic microenvironment by expressing a hypoxia-inducible heterodimeric transcription factor, hypoxia-inducible factor- $1 \alpha(\mathrm{HIF}-1 \alpha)$, which serves an essential role in sensing and responding to hypoxia-induced alterations 
in the cellular environment $(10,11)$. HIF-1 $\alpha$ regulates numerous hypoxia-responsive genes which are involved in a variety of biological functions. Under normoxic conditions, HIF-1 $\alpha$ is targeted for proteasomal degradation by an iron-containing prolyl hydroxylase (PHD) enzyme (12). By contrast, under hypoxic conditions, HIF-1 $\alpha$ evades hydroxylation and translocates to the nucleus where it heterodimerizes with HIF-1 $\beta$ (13). Previous studies have demonstrated that HIF-1 $\alpha$ signaling pathway-mediated tissue hypoxia serves a fundamental role in the regulation of stem/progenitor cell recruitment, and the retention and differentiation of regenerating tissues $(14,15)$. It has also been demonstrated that HIF-1 $\alpha$ mediates the angiogenic, and osteogenic phases of bone repair and regeneration, via genetic or pharmacological means $(10,16)$.

Deferoxamine (DFO) iron chelators can artificially induce hypoxia by inhibiting PHD activity via chelation of iron in the PHD active sites, which is required for PHD function, including stabilization of HIF-1 $\alpha$ expression $(11,17)$. Therefore, DFO has been extensively used as a hypoxia-mimetic agent in normoxia and the effects are comparable to those resulting from reduced atmospheric oxygen levels (17). Previous studies have demonstrated that DFO directly triggers the differentiation of marrow stromal cells (MSCs) (18), and increases bone formation by stimulating osteogenesis and angiogenesis through stabilization of HIF-1 $\alpha$ expression (10).

Based on these observations, the influence of DFO on hPDLCs was investigated and the preconditioning effect of DFO on hPDLC osteogenic differentiation was evaluated. The results of the present study may contribute to the efforts to design a cell therapy for periodontal disease.

\section{Materials and methods}

Cell culture. All the participants of the present study signed informed consent forms and the experimental protocol was approved by the local Ethical Committee of The Second Affiliated Hospital of Harbin Medical University (Harbin, China). All hPDLCs were derived from PDL tissue which was obtained from a total of 10 clinically healthy premolar teeth extracted for orthodontic reasons (two males and three females aged 12-16 years) from April to June 2015 at The Second Affiliated Hospital of Harbin Medical University.

The extracted teeth were washed three times with PBS Periodontal tissue was obtained from the middle third of the root using a sterile scalpel. The tissue was cut with scissors into small fragments following rinsing with growth media ( $\alpha$-Minimum Essential Medium; Invitrogen; Thermo Fisher Scientific, Inc., Waltham, MA, USA) and placed in $25-\mathrm{cm}^{2}$ culture flasks containing Dulbecco's modified Eagle's medium (DMEM; Gibco; Thermo Fisher Scientific, Inc.) supplemented with $10 \%(\mathrm{v} / \mathrm{v})$ fetal bovine serum (FBS; GE Healthcare Life Sciences, Logan, UT, USA), 4 mM L-glutamine, $100 \mathrm{U} / \mathrm{ml}$ penicillin and $100 \mu \mathrm{g} / \mathrm{ml}$ streptomycin. Peridontal tissue was incubated at $37^{\circ} \mathrm{C}$ in a humidified atmosphere containing $5 \%$ carbon dioxide and $95 \%$ air. PDLCs were passaged until they reached $80 \%$ confluence and the cells from the third to fifth passages were used for the subsequent experiments.

For hypoxia treatment, hPDLCs were seeded on plates and incubated with various concentrations $(0,5,10$ and $20 \mu \mathrm{M})$ of DFO (Sigma-Aldrich; Merck KGaA, Darmstadt, Germany).
Cell viability assay. The cell viability of hPDLCs was measured using an MTT assay. hPDLCs were seeded in 96-well plates at an initial density of $3.0 \times 10^{3}$ cells/well and cultured in DMEM with $10 \%$ FBS for $24 \mathrm{~h}$. Subsequently, hPDLCs were starved overnight by changing DMEM to a serum-free medium to compensate for the effect of growth factors, and then incubated with $0,5,10$ and $20 \mu \mathrm{M}$ DFO for 24,48 or $72 \mathrm{~h}$. At each time point, $20 \mu \mathrm{l}$ MTT $(0.5 \mathrm{mg} / \mathrm{ml}$ final concentration; Sigma-Aldrich; Merck KGaA) solution was added into each well and hPDLCs were incubated at $37^{\circ} \mathrm{C}$ for $4 \mathrm{~h}$. The supernatants were subsequently removed and replaced with $150 \mu \mathrm{l}$ dimethyl sulfoxide (Sigma-Aldrich; Merck KGaA) for $10 \mathrm{~min}$ to solubilize the formazan. The absorbance was measured at a wavelength of $490 \mathrm{~nm}$ with a microplate reader (Model 550; Bio-Rad Laboratories, Inc., Hercules, CA, USA). A total of three independent experiments were performed.

Reverse transcription-quantitative polymerase chain reaction $(R T-q P C R)$. To quantify the mRNA expression of HIF-1 $\alpha$, runt-related transcription factor 2 (Runx-2), osteopontin (OPN), collagen type I (Col-1), $\alpha$-smooth muscle actin ( $\alpha$-SMA) and transforming growth factor- $\beta 1$ (TGF- $\beta 1)$, confluent cells were incubated with $\operatorname{DFO}(0,5,10$ or $20 \mu \mathrm{M})$ for 2 days and total RNA was isolated with TRIzol (Invitrogen; Thermo Fisher Scientific, Inc.), according to the manufacturer's protocol. The cDNA was synthesized from $1 \mu \mathrm{g}$ total RNA using the PrimeScript ${ }^{\mathrm{TM}}$ RT Reagent kit (cat. no. RR047A; Takara Biotechnology Co. Ltd., Dalian, China), according to the manufacturer's protocol. The expression of HIF-1 $\alpha$ was detected using qPCR using SYBR ${ }^{\circledR}$ Premix Ex Taq ${ }^{\mathrm{TM}}$ II (cat. no. RR820A; Takara Biotechnology Co. Ltd.). The Mx3005P qPCR System Thermal Cycler (Agilent Technologies, Inc., Santa Clara, USA) was used according to the manufacturer's protocol. A total of $1 \mu \mathrm{l}$ cDNA was used as a template in a $25-\mu 1$ reaction mixture. The mixture was initially heated at $95^{\circ} \mathrm{C}$ for $30 \mathrm{sec}$ followed by 40 cycles of denaturation at $95^{\circ} \mathrm{C}$ for $5 \mathrm{sec}$ and combined annealing/extension at $60^{\circ} \mathrm{C}$ for $30 \mathrm{sec}$. Quantities of the reaction products were normalized to the $\beta$-actin reference gene. All reactions were run in triplicate. The data was analyzed using the comparative threshold cycle $\left(2^{-\Delta \Delta C q}\right)$ method (19). The primer sequences used for all qPCR reactions are listed in Table I.

Wound healing assay. The scratch-wound assay was performed to determine the effects of DFO on the migration of hPDLCs. The hPDLCs were seeded into 6-well plates at an initial density of $5 \times 10^{5}$ cells/well and cultured to reach $80-90 \%$ confluence as a monolayer. In order capture images of the corresponding locations at each indicated time point, the outside surface of each well was marked prior to the test. The surface of the cellular monolayer was then gently and linearly scratched with a sterile pipette tip. Following washing and removal of the detached cells, the plates were incubated at $37^{\circ} \mathrm{C}$ with fresh medium containing 0,5, 10 and $20 \mu \mathrm{M}$ DFO. Following 0, 24 and $48 \mathrm{~h}$ of incubation, phase contrast images of the wounds were visualized under a light microscope (Eclipse 80i; Nikon Corporation, Tokyo, Japan) and recorded using NIS-Elements Basic Research software (Nikon Corporation). The number of hPDLCs that migrated into the scratch area was counted to quantify the cell migration rates. Experiments were independently repeated three times. 
Table I. Primer sequences for quantitative polymerase chain reaction.

Primer sequence $\left(5^{\prime}-3^{\prime}\right)$

\begin{tabular}{lll}
\cline { 2 - 3 } Gene & \multicolumn{1}{c}{ Forward } & \multicolumn{1}{c}{ Reverse } \\
\hline HIF-1 $\alpha$ & GTGTTATCTGTCGCTTTGAGTC & CTGGCTGCTGTAATAATGTTCC \\
$\alpha$-SMA & CGGGACATCAAGGAGAAACT & CCATCAGGCAACTCGTAACTC \\
TGF- $\beta 1$ & ACCTTGGGCACTGTTGAAGT & CTCTGGGCTTGTTTCCTCAC \\
Runx 2 & TAGGCGCATTTCAGGTGCTT & GGTGTGGTAGTGAGTGGTGG \\
OPN & AGCAGCTTTACAACAAATACCCAG & TTACTTGGAAGGGTCTGTGGG \\
Col- 1 & TGAACTTGTTGCTGAGGGC & GCAGGCGTGATGGCTTAT \\
$\beta$-actin & TTGCCGACAGGATGCAGAA & CTCCTGCTTGCTGATCCACAT
\end{tabular}

HIF- $1 \alpha$, hypoxia-inducible factor $1-\alpha$; $\alpha$-SMA, $\alpha$-smooth muscle actin; TGF- $\beta 1$, transforming growth factor- $\beta 1$; Runx 2 , runt-related transcription factor 2; OPN, osteopontin; Col-1, collagen type 1.

Osteogenic induction. Cells were seeded in 6-well plates at a density of $7 \times 10^{4}$ cells/well. When confluent, the cells were treated with $0,5,10$ or $20 \mu \mathrm{M}$ DFO for 2 days, and then changed to the osteogenic medium DMEM supplemented with $10 \%$ FBS, $10 \mathrm{mM}$ sodium $\beta$-glycerophosphate, $100 \mathrm{nM}$ dexamethasone and $50 \mathrm{mg} / \mathrm{l}$ ascorbic acid, to induce mineralization. Osteogenic medium was replaced every 3 days.

Alkaline phosphatase activity assay. Following 5 days of mineralization, ALP activity was measured using a p-nitrophenol phosphate assay (ALP assay kit; Beyotime Institute of Biotechnology, Shanghai, China), according to the manufacturer's protocol. Initially, hPDLCs were washed three times with PBS and lyzed with $0.1 \%$ Triton X-100. Subsequently, the supernatant was collected into a 96 -well plate, mixed with the substrates and p-nitrophenol from the ALP assay kit and incubated for $10 \mathrm{~min}$ at $37^{\circ} \mathrm{C}$. Following the incubation, the absorbance of p-nitrophenol was measured using a microplate reader at a wavelength of $405 \mathrm{~nm}$.

Immunofluorescence analysis. Immunofluorescence analysis of Col-1 in hPDLCs was performed on the 5th day of osteogenic induction. Cells were fixed in $4 \%$ paraformaldehyde at room temperature for $30 \mathrm{~min}$. Following rinsing with PBS, cells were incubated with $3 \%$ bovine serum albumin (Beyotime Institute of Biotechnology) for $1 \mathrm{~h}$ at room temperature to bind nonspecific binding sites. Cells were then incubated overnight at $4^{\circ} \mathrm{C}$ with a rabbit anti-human Col-I polyclonal primary antibody (1:500; cat. no. ab34710; Abcam, Cambridge, UK). The fluorescein isothiocyanate-conjugated goat polyclonal anti-rabbit immunoglobulin $\mathrm{G}$ was used as the secondary antibody (1:160; cat. no. ZF-0314; OriGene Technologies, Inc., Rockville, MD, USA) and incubated with the membrane for $1 \mathrm{~h}$ at room temperature. Then, the samples were incubated with DAPI (Sigma-Aldrich; Merck KGaA) for 3 min at room temperature to allow for nucleus detection. Cell staining was observed using a fluorescence microscope (Nikon Corporation). Fluorescence quantification was achieved by comparing the integrated optical density/pixel value of Col-1 between the DFO group and the control group using ImageJ software (version 1.42q; National Institutes of Health, Bethesda, MD, USA).
Alizarin red $S$ (ARS) staining. Following induction of mineralization for 14 days, mineral nodule formation was determined by $0.1 \%$ ARS staining (pH 4.2; Sigma-Aldrich; Merck KGaA). For each experiment, human hPDLC monolayers were washed twice with PBS and fixed with $95 \%$ ice-cold ethanol for $10 \mathrm{~min}$ and rinsed twice with distilled water. Fixed cells were stained with ARS for $30 \mathrm{~min}$ at room temperature and washed with distilled water once more. ARS staining images were captured using a phase-contrast microscope and calculated using the Matlab image analysis software (MathWorks, Natick, MA, USA).

Statistical analysis. Statistical analysis was performed using SPSS software for Windows (version 19.0.1; IBM Corp., Armonk, NY, USA). All experiments were performed in triplicate and results were expressed as the mean \pm standard deviation. Statistical analysis was performed by one-way analysis of variance followed by the Dunnett's test. $\mathrm{P}<0.05$ was considered to indicate a statistically significant difference.

\section{Results}

Effects of DFO on cell viability. MTT assays demonstrated a concentration-dependent effect of DFO on hPDLC viability (Fig. 1A). Following a $48 \mathrm{~h}$ treatment, no significant difference was observed between the 5 and $10 \mu \mathrm{M}$ DFO groups compared with the control group. At $72 \mathrm{~h}$, the viability of hPDLCs decreased in all DFO groups compared with the control group (all $\mathrm{P}<0.05$ ). When treated with high concentration of DFO $(20 \mu \mathrm{M})$, the viability of hPDLC significantly decreased as early as after $24 \mathrm{~h}$.

DFO promotes the expression of HIF-1 $\alpha$ in hPDLCs. As presented in Fig. 1B, the expression of HIF-1 $\alpha$ mRNA in hPDLCs was increased by DFO. Compared with the control group, at the $48 \mathrm{~h}$ time point, the expression levels of HIF-1 $\alpha$ mRNA were increased 1.4-fold and 1.6-fold following incubation with 5 and $10 \mu \mathrm{M}$ DFO, respectively (both $\mathrm{P}<0.05$ ).

Scratch wound healing assay. The results of the scratch-wound assay are presented in Fig. 2A. On the 2nd day DFO 


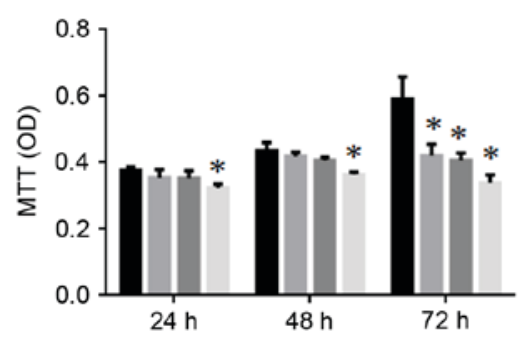

B

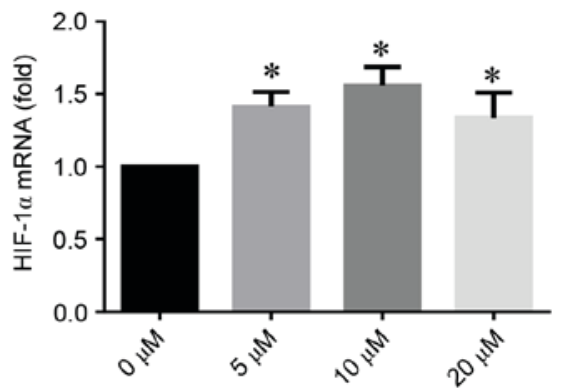

Figure 1. Effects of DFO on hPDLCs viability and mRNA expression of HIF-1 $\alpha$. (A) Cellular viability assayed by MTT. Histograms depict a concentration-dependent decrease in hPDLC proliferation following treatment with different concentrations of DFO $(0,5,10$ and $20 \mu \mathrm{M})$. (B) Relative gene expression values for HIF-1 $\alpha$ evaluated following hPDLC stimulation with DFO for 2 days, using the quantitative polymerase chain reaction. "P $<0.05$ vs. the respective control group. hPDLCs, human periodontal ligament cells; OD, optical density; DFO, deferoxamine; HIF-1 $\alpha$, hypoxia-inducible factor-1 $\alpha$.

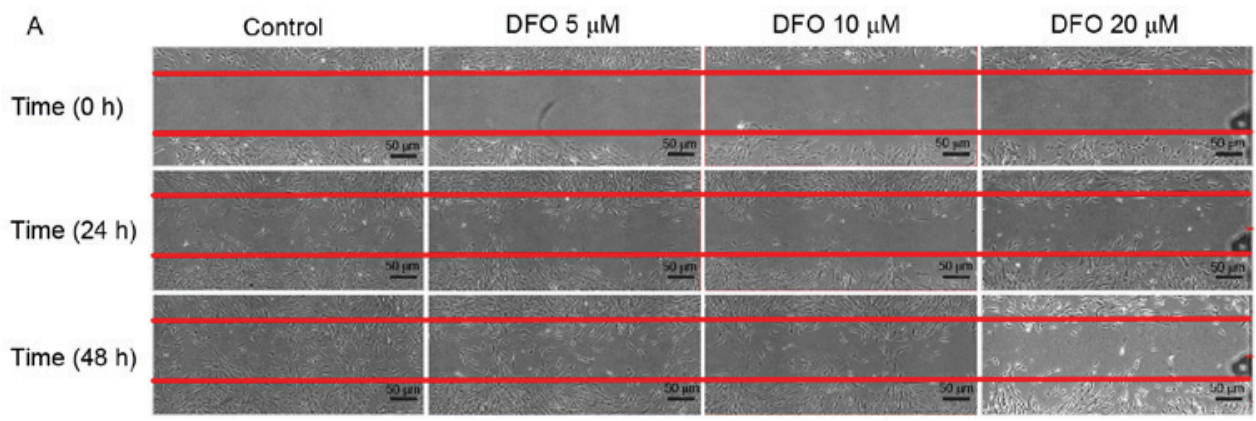

B

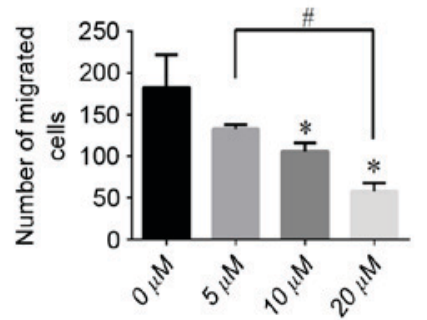

C

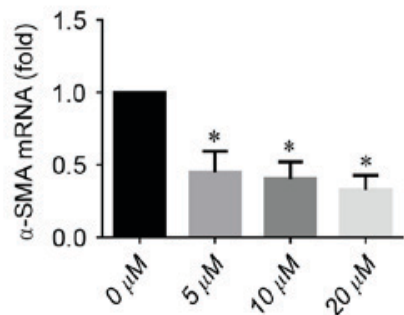

D

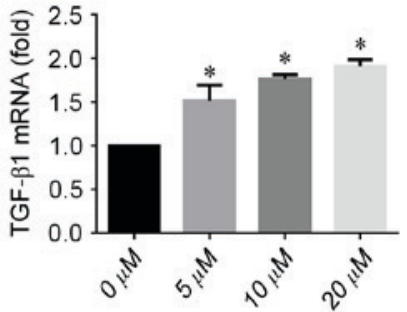

Figure 2. Effects of DFO on cell migration and mRNA expression of $\alpha$-SMA and TGF- $\beta 1$. (A) Wound healing migration assay of DFO-treated and untreated human periodontal ligament cells. (B) Quantitative analysis of the number of cells crossing the edge of wound at $48 \mathrm{~h}$ following introduction of the scratch (C) $\alpha$-SMA RNA expression $48 \mathrm{~h}$ following treatment with DFO compared with the control group. (D) TGF- $\beta 1$ RNA expression $48 \mathrm{~h}$ following treatment with DFO compared with the control group. " $\mathrm{P}<0.05$ vs. the control group; ${ }^{*} \mathrm{P}<0.05$. $\alpha$-SMA, $\alpha$-smooth muscle actin; TGF- $\beta 1$, transforming growth factor- $\beta 1$; HIF-1 $\alpha$, hypoxia-inducible factor- $1 \alpha$; DFO, deferoxamine.

demonstrated an inhibitory effect on hPDLC migration in a concentration-dependent manner. There was no significant difference in the number of cells that migrated into the wound area between the low concentration $(5 \mu \mathrm{M})$ DFO group and the control group (Fig. 2B). In the $20-\mu \mathrm{M}$ DFO group, an increased amount of interspersed non-viable cells could be observed under the light microscope, compared with the control group. Subsequently, the $\alpha$-SMA and TGF- $\beta 1$ mRNA expression during scratch wound healing was examined. Results from qPCR demonstrated that DFO downregulated the expression of $\alpha$-SMA (Fig. 2 C). Contrastingly, the level of TGF- $\beta 1$ mRNA was upregulated by DFO in a concentration-dependent manner (all $\mathrm{P}<0.05$; Fig. 2D).

\section{Effects of DFO on osteogenic differentiation}

ALP activity assay. ALP activity assays demonstrated that DFO significantly promoted ALP activity in hPDLCs.
Particularly, $5 \mu \mathrm{M}$ DFO demonstrated an increased activating potential compared with the control group $(\mathrm{P}<0.05$; Fig. 3A).

DFO induces the expression of osteogenic-associated genes in vitro. qPCR demonstrated that DFO upregulated the mRNA expression of hPDLC-osteogenic markers after 5 days of mineralization (Fig. 3B-D). Similarly to the results of the ALP assay, $5 \mu \mathrm{M}$ DFO exhibited an increased ability to stimulate osteogenic differentiation compared with $10 \mu \mathrm{M}$ DFO. Compared with the control group, the mRNA expression of Runx2 increased to nearly 3.7- and 2.3-fold when hPDLCs were treated with 5 and $10 \mu \mathrm{M}$ DFO, respectively (Fig. 3B). The expression level of OPN increased to $>5$ - and 3 -fold when treated with 5 and $10 \mu \mathrm{M}$ DFO, compared with the control group (Fig. 3C). The Col-1 expression quadrupled and doubled compared with the control group when 5 and $10 \mu \mathrm{M}$ DFO 


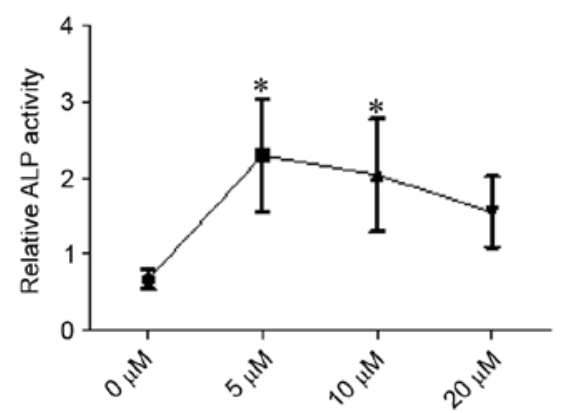

C

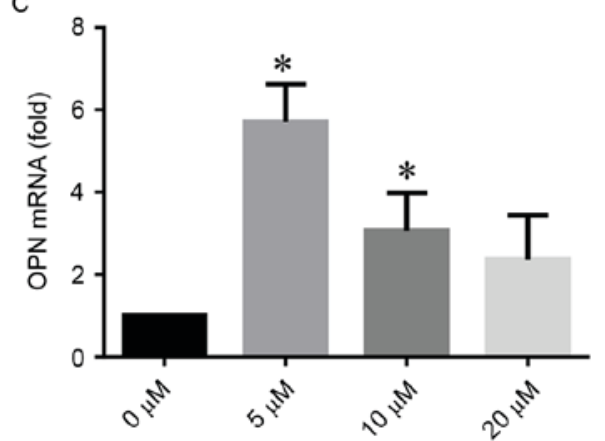

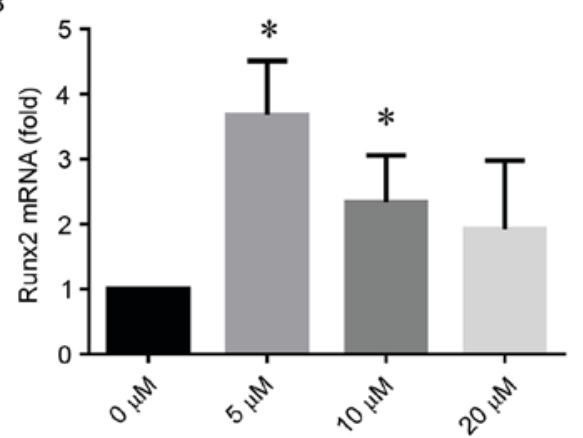

D

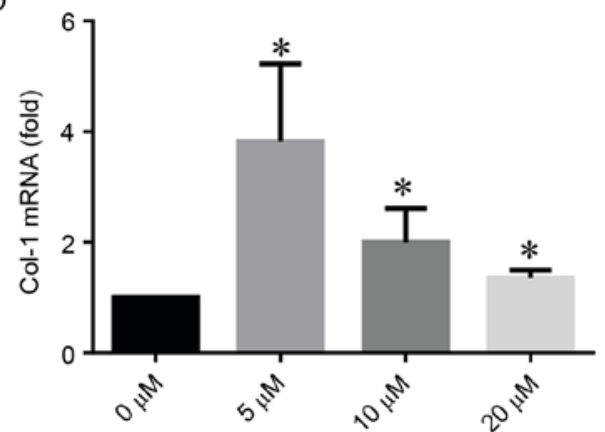

Figure 3. ALP activity assay and expressions of osteogenic-marker genes following induction of mineralization. (A) Quantitative ALP assay. (B) mRNA expressions of Runx2 following treatment with DFO. (C) mRNA expressions of OPN following treatment with DFO. (D) mRNA expressions of Col-1 following treatment with DFO. "P<0.05 vs. the control group. Runx2, runt-related transcription factor 2; OPN, osteopontin; Col-1, collagen type I; ALP, alkaline phosphatase; DFO, deferoxamine.

were applied, respectively (Fig. 3D). The $20 \mu \mathrm{M}$ treatment with DFO also induced significant changes in Col-1 expression $(\mathrm{P}<0.05)$.

DFO increases Col-1 synthesis in hPDLCs. Immunofluorescence staining for Col-1 demonstrated a significant increase in staining intensity following treatment with 5 and $10 \mu \mathrm{M}$ DFO, compared with the control group (both $\mathrm{P}<0.05$; Fig. 4).

ARS staining. Mineralized nodule formation was analyzed using ARS staining following 14 days of osteogenic induction (Fig. 5). The results were consistent with the data obtained from the ALP activity assay and qPCR. There was an increased calcium deposition in DFO groups compared with the control group, and the intensity of ARS staining with 5 and $10 \mu \mathrm{M}$ DFO was increased 2.977 and 2.562-fold compared with the control group, respectively. However, due to low cell viability, no mineralization was observed when hPDLCs were treated with $20 \mu \mathrm{M}$ DFO (data not shown).

\section{Discussion}

Periodontitis is one of the most common inflammatory diseases resulting from a complex interplay between bacterial infection and host responses, and is characterized by destruction of the attachment apparatus and the supporting bone of teeth (20). Periodontitis is the most prevalent cause of tooth loss and is difficult to heal (20). Searching for an effective method for periodontium remodeling is an important part of the effort to develop periodontitis therapeutic strategies.
PDL cells form a heterogeneous cell population and have a potential to regenerate periodontal tissue under certain conditions (4). Previous in vitro and in vivo studies demonstrated that under hypoxic conditions the proportion of osteogenesis cells and their differentiation potential are increased compared with under normoxic conditions $(9,21)$. In the present study, genetics-based evidence that the DFO-induced hypoxia has the potency to induce osteogenic differentiation in hPDLCs was presented.

Initially, the effect of DFO on human PDL cell viability was investigated using the MTT method. It was demonstrated that proliferation of hPDLCs was inhibited by DFO in a concentration-dependent manner, while low concentrations of DFO exhibited no apparent inhibitory effect on hPDLCs. One of the reasons for the negative effect of DFO on hPDLC proliferation may be associated with the removal of iron, which serves a role in metabolic pathways. It has been previously suggested that through chelating the intracellular iron pool, DFO can inhibit the activity of ribonucleotide reductase, which is required for the synthesis of deoxynucleotides and results in G1/S cell-cycle arrest (22).

The MTT results were consistent with those previously reported in the literature. The results demonstrated that DFO-induced hypoxia could downregulate the growth of osteoblast through inhibition of Wnt signaling pathways via activation of HIF-1 $\alpha$ (23). The proliferation of umbilical cord-derived human mesenchymal stem cells is also significantly inhibited by DFO through influencing the cell cycle, attenuating the intercellular signal transmission and altering cell morphology/ultrastructure (24). Previous experimental in vivo and in vitro research, as well as several initial clinical 

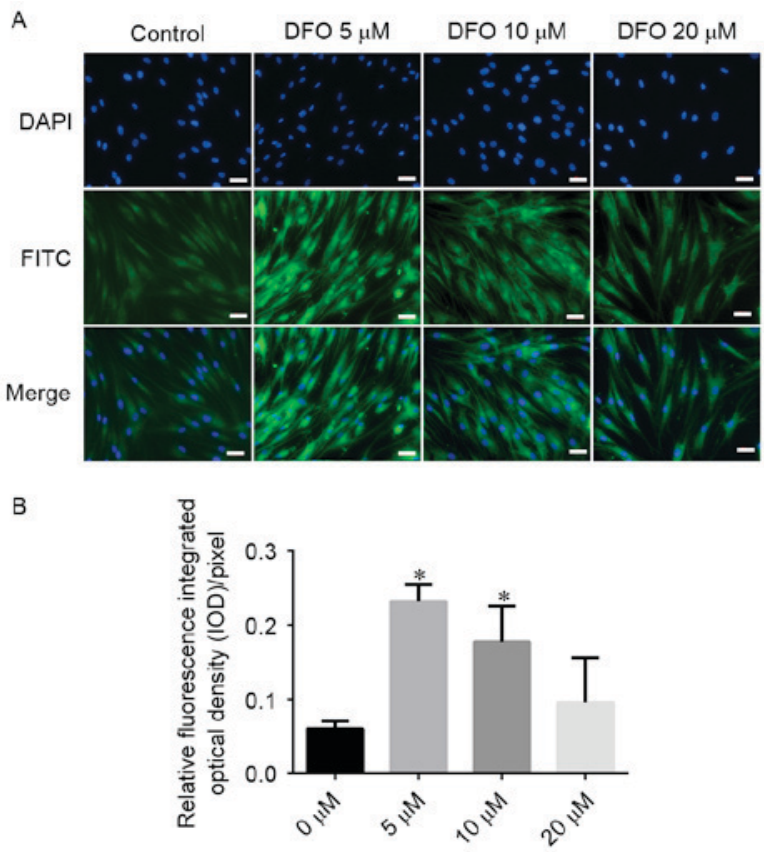

Figure 4. Immunofluorescent analysis of Col-1 using FITC in hPDLCs treated with DFO. (A) hPDLCs were incubated with anti-Col-1 antibody (green), and nuclei were stained with DAPI (blue). Scale bar, $200 \mu \mathrm{m}$. (B) Fluorescence quantification analysis performed by comparing the IOD/pixel value of Col-1 in DFO with the control group. " $\mathrm{P}<0.05$ vs. the control group. Col- 1 , collagen type 1; FITC, fluorescein isothiocyanate; hDPLCs, human periodontal ligament cells; IOD, integrated optical density; DFO, deferoxamine.

trials, demonstrated that DFO could effectively inhibit certain neoplasm growths and induce cell differentiation (25). Additionally, certain experiments demonstrated that inhibition of growth is an important transition point in the initiation of differentiation and mineralization of proliferating cells (26). The mechanism of DFO activity on PDL cells is complex and it is unclear whether hPDLC proliferation inhibition is associated with differentiation.

The migration of hPDLCs towards the site of injury is necessary for periodontium healing. In the present study, scratch-wound assays were performed to observe the effects of DFO on the migration of hPDLCs. The results indicated that low concentrations of DFO exhibited little inhibition on migration within $48 \mathrm{~h}$; however, the high concentration exhibited a concentration-dependent inhibitory effect. In agreement with the present study, a previous study reported that stress-fiber formation was inhibited through cellular iron depletion mediated by DFO, which could lead to inhibition of cellular migration (27). A previous study on the inhibitory effect of DFO was also performed in the context of anticancer treatments (28).

The mRNA expression of $\alpha$-SMA and TGF- $\beta 1$, which are involved in scratch wound healing, was also examined. In wound contraction, $\alpha$-SMA serves a crucial role by increasing contractile activity of stress fibers and the abundance of this protein is positively associated with wound contraction efficiency in vivo (29). The results from qPCR demonstrated that the expression of $\alpha$-SMA was downregulated by DFO in a concentration-dependent manner, consistent with the wound healing assay results. Downregulated expression of $\alpha$-SMA may be partly due to a low cellular oxygen tension in hPDLCs resulting from upregulated expression of HIF-1 $\alpha$ induced
A
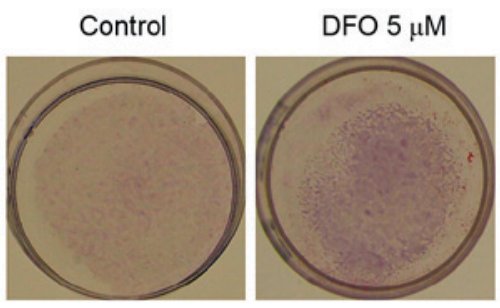

DFO $10 \mu \mathrm{M}$

B
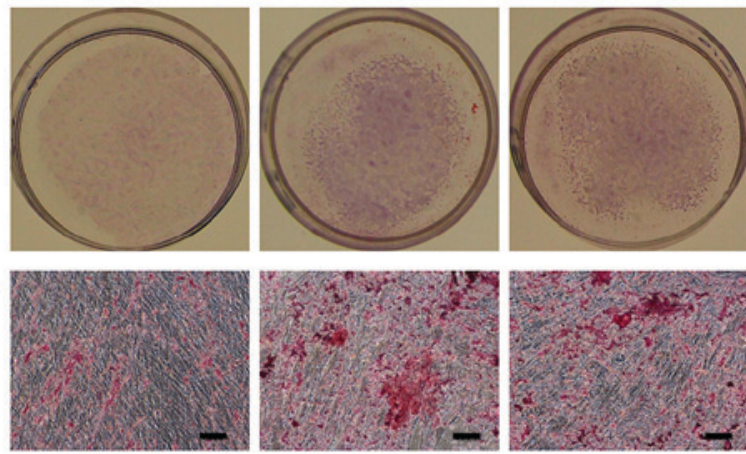

C

\#
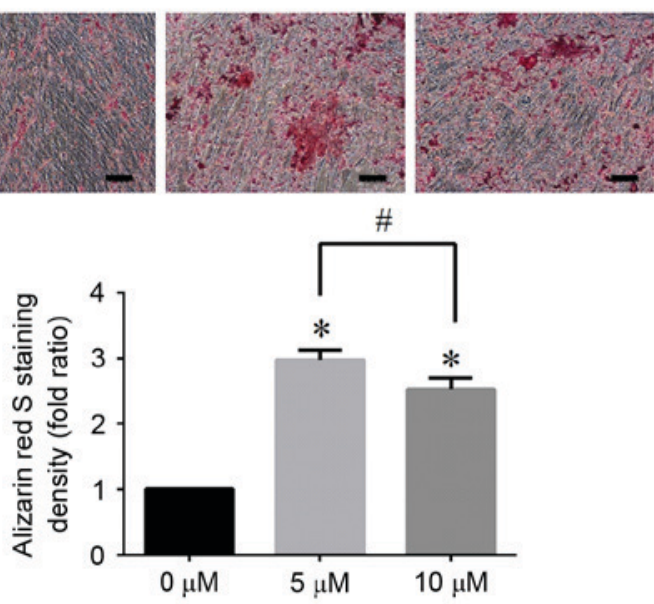

Figure 5. Mineral deposition assayed by ARS staining. (A) Whole cell-culture dishes. (B) Mineralized deposition in hPDLCs. Scale bar, $250 \mathrm{~mm}$. (C) The ratio of ARS staining density compared with the control. " $\mathrm{P}<0.05$ vs. control group; ${ }^{\text {P }}<0.05$. ARS, alizarin red $\mathrm{S}$; hPDLCs, human periodontal ligament cells; DFO, deferoxamine.

by DFO. Previous studies have demonstrated that reduction of extra- and intra-cellular tension leads to the removal of $\alpha$-SMA from stress fibers within h; under continued low stress, expression levels of $\alpha$-SMA are reduced $(29,30)$. The results of the present study were in agreement with previous evidence that dermal fibroblasts exhibit low expression of $\alpha$-SMA, and decreased migration and proliferation under hypoxic conditions (31). In the future, DFO may serve a role in the treatment of liver fibrosis by decreasing the expression of $\alpha$-SMA in hepatic stellate cells (32).

TGF- $\beta 1$, which is highly expressed in the entirety of periodontal ligament tissue, is a multifunctional cytokine that regulates various cellular activities, including cell differentiation, synthesis of extracellular matrix proteins and wound repair $(33,34)$. TGF- $\beta 1$ induces $\alpha$-SMA expression in myofibroblasts and is a key regulator of type I collagen (25). In the present study, the expression level of TGF- $\beta 1$ in DFO-treated hPDLCs increased markedly, which is consistent with the previous experiments demonstrating increased levels of active and total TGF- $\beta 1$ in response to hypoxic conditions $(30,35)$. $\alpha$-SMA expression decreased in hypoxic conditions, suggesting that hypoxia desensitized the expression of $\alpha$-SMA in the presence of TGF- $\beta 1$. This phenomenon may be in part due to concomitant downregulation of the expression of the TGF- $\beta 1$ receptor subunit, TGF $\beta$-RII, possibly by a negative TGF- $\beta 1$ feedback loop (30). This mechanism was proposed in a previous study, which indicated significantly reduced expression of $\alpha$-SMA and wound contraction despite a high level of TGF- $\beta 1$ when skin myofibroblasts were cultured in hypoxic condition for 5 days (30). In addition, an increased level of TGF- $\beta 1$ could further increase the expression of HIF-1 $\alpha$ (36) 
and stabilize hypoxia in hPDLCs. In the future, thorough studies focused on this issue shall be conducted.

Hard tissue regeneration is the most important part in periodontium reconstruction. It is accepted that hPDLCs demonstrate specific osteoblast-like properties, have osteogenic characteristics (37), and can differentiate into cementoblasts and osteoblasts, which are necessary for cementum and alveolar bone formation, respectively. The present study revealed that preconditioning of hPDLCs with DFO could significantly stimulate ALP activity, upregulate the expression of osteogenesis/cementogenesis-associated genes and induce mineralization.

ALP, a membrane-associated enzyme, serves a key role in connective tissue calcification and mineral deposition (38). Furthermore, ALP is also an early marker for osteoblastic differentiation, and an indicator of the presence of osteoblasts and new bone formation (39). HPDLCs exhibit increased ALP activity compared with gingival fibroblasts, suggesting that hPDLCs are crucial in the development of osteogenic characteristics (38). In the present study, DFO significantly increased ALP activity in hPDLCs and $5 \mu \mathrm{M}$ DFO had the most prominent effect.

Runx2, OPN and Col-1 are osteogenic-specific genes in hPDLCs. Runx2, the osteoblast-specific transcription factor, is described as the master regulator in the commitment of osteoblastogenesis (40) and regulates the expression of a number of genes associated with osteoblasts (41). OPN produced by osteoblasts at various stages of differentiation is one of the vital non-collagenous bone matrix proteins. High OPN calcium-binding potential can promote calcium adhesion, thus influencing mineralization events (42). Col-1, an early marker of osteogenic differentiation, is a major extracellular matrix component of periodontal tissues that promotes osteoblast differentiation and mineralization (43). The qPCR conducted in the present study further demonstrated that DFO could enhance osteogenic differentiation of hPDLCs by markedly accelerating the expression of the osteogenic-specific genes.

To verify the role of DFO in the mineralization of hPDLCs, a mineralization assay using ARS staining was performed. An enhanced calcium deposition was observed in DFO groups compared with the control group, indicating that DFO could promote the mineralization propertied of hPDLCs.

From the qPCR and ARS staining results it was inferred that $5 \mu \mathrm{M}$ DFO is more efficient than $10 \mu \mathrm{M}$ for mineralization induction under normoxic condition. The adverse effect of high DFO level on osteogenic ability was partly due to an excessive depletion of iron in hPDLCs, which could also lead to decreased cell viability. These results reinforce the previous findings that osteogenesis is dependent on iron homeostasis in a biphasic manner: Mild iron deficiency promotes osteogenesis, while severe iron deficiency can inhibit this process (44). The results are further reinforced by the evidence that a higher concentration of $20 \mu \mathrm{M}$ DFO completely abolished mineralization due to its toxicity on hPDLCs. Therefore, it is vital to determine a suitable DFO concentration in order to achieve the optimal osteogenic efficacy.

To conclude, the data gathered in the present study indicate that by affecting osteogenic-associated factors, DFO could enhance osteogenic differentiation and mineralization in hPDLCs. These results provide a reference for further research on the molecular mechanisms behind these processes. The results may also have clinical implications since preconditioning of hPDLCs with DFO may improve the efficacy of cell therapy and DFO is a promising candidate for improving hard tissue regenerative potential in periodontal disease. The mechanism underlying periodontal hard tissue formation by hPDLCs treated with DFO in vivo remains to be elucidated.

\section{Acknowledgements}

The present study was supported by the National Natural Science Foundation of China (grant no. 81570951) and the Special Foundation for Sino-Russian Translational Medicine Research Center of Harbin Medical University (grant no. CR201412).

\section{References}

1. Chai Y, Jiang X, Ito Y, Bringas P Jr, Han J, Rowitch DH, Soriano P, McMahon AP and Sucov HM: Fate of the mammalian cranial neural crest during tooth and mandibular morphogenesis. Development 127: 1671-1679, 2000.

2. Beertsen W, McCulloch Ca and Sodek J: The periodontal ligament: A unique, multifunctional connective tissue. Periodontol 2000 13: 20-40, 1997.

3. Alvarez R, Lee HL, Wang CY and Hong C: Characterization of the osteogenic potential of mesenchymal stem cells from human periodontal ligament based on cell surface markers. Int J Oral Sci 7: 213-219, 2015.

4. Seo BM, Miura M, Gronthos S, Bartold PM, Batouli S, Brahim J, Young M, Robey PG, Wang CY and Shi S: Investigation of multipotent postnatal stem cells from human periodontal ligament. Lancet 364: 149-155, 2004.

5. Semenza GL: Hypoxia-inducible factor 1: Oxygen homeostasis and disease pathophysiology. Trends Mol Med 7: 345-350, 2001.

6. Harris AL: Hypoxia-a key regulatory factor in tumour growth. Nat Rev Cancer 2: 38-47, 2002.

7. Eltzschig HK and Carmeliet P: Hypoxia and inflammation. N Engl J Med 364: 656-665, 2011.

8. Simon MC and Keith B: The role of oxygen availability in embryonic development and stem cell function. Nat Rev Mol Cell Biol 9: 285-296, 2008

9. Ren H, Cao Y, Zhao Q, Li J, Zhou C, Liao L, Jia M, Zhao Q, Cai H, Han ZC, et al: Proliferation and differentiation of bone marrow stromal cells under hypoxic conditions. Biochem Biophys Res Commun 347: 12-21, 2006.

10. Wan C, Gilbert SR, Wang Y, Cao X, Shen X, Ramaswamy G, Jacobsen KA, Alaql ZS, Eberhardt AW, Gerstenfeld LC, et al: Activation of the hypoxia-inducible factor-1 pathway accelerates bone regeneration. Proc Natl Acad Sci USA 105: 686-691, 2008.

11. Ivan M, Kondo K, Yang H, Kim W, Valiando J, Ohh M, Salic A, Asara JM, Lane WS and Kaelin WG Jr: HIFalpha targeted for VHL-mediated destruction by proline hydroxylation: Implications for O2 sensing. Science 292: 464-468, 2001.

12. Semenza GL: HIF-1: Mediator of physiological and pathophysiological responses to hypoxia. J Appl Physiol (1985) 88: $1474-1480,2000$

13. Bruick RK and McKnight SL: A conserved family of prolyl-4-hydroxylases that modify HIF. Science 294: 1337-1340, 2001.

14. Ceradini DJ, Kulkarni AR, Callaghan MJ, Tepper OM, Bastidas N, Kleinman ME, Capla JM, Galiano RD, Levine JP and Gurtner GC: Progenitor cell trafficking is regulated by hypoxic gradients through HIF-1 induction of SDF-1. Nat Med 10: 858-864, 2004

15. Zhao T, Zhang CP, Liu ZH, Wu LY, Huang X, Wu HT, Xiong L, Wang X, Wang XM, Zhu LL and Fan M: Hypoxia-driven proliferation of embryonic neural stem/progenitor cells-role of hypoxia-inducible transcription factor-1alpha. FEBS J 275: 1824-1834, 2008.

16. Wang Y, Wan C, Deng L, Liu X, Cao X, Gilbert SR, Bouxsein ML, Faugere MC, Guldberg RE, Gerstenfeld LC, et al: The hypoxia-inducible factor alpha pathway couples angiogenesis to osteogenesis during skeletal development. J Clin Invest 117: 1616-1626, 2007. 
17. Hirsilä M, Koivunen P, Xu L, Seeley T, Kivirikko KI and Myllyharju J: Effect of desferrioxamine and metals on the hydroxylases in the oxygen sensing pathway. FASEB J 19: 1308-1310, 2005.

18. Qu ZH, Zhang XL, Tang TT and Dai KR: Promotion of osteogenesis through beta-catenin signaling by desferrioxamine. Biochem Biophys Res Commun 370: 332-337, 2008.

19. Livak KJ and Schmittgen TD: Analysis of relative gene expression data using real-time quantitative PCR and the 2(-Delta Delta C(T)) method. Methods 25: 402-408, 2001.

20. Pihlstrom BL, Michalowicz BS and Johnson NW: Periodontal diseases. Lancet 366: 1809-1820, 2005.

21. Lennon DP, Edmison JM and Caplan AI: Cultivation of rat marrow-derived mesenchymal stem cells in reduced oxygen tension: Effects on in vitro and in vivo osteochondrogenesis. J Cell Physiol 187: 345-355, 2001.

22. Nurtjahja-Tjendraputra E, Fu D, Phang JM and Richardson DR: Iron chelation regulates cyclin D1 expression via the proteasome: A link to iron deficiency-mediated growth suppression. Blood 109: 4045-4054, 2007.

23. Chen D, Li Y, Zhou Z, Wu C, Xing Y, Zou X, Tian W and Zhang C: HIF-1 $\alpha$ inhibits Wnt signaling pathway by activating Sost expression in osteoblasts. PLoS One 8: e65940, 2013.

24. Zeng HL, Zhong Q, Qin YL, Bu QQ, Han XA, Jia HT and Liu HW: Hypoxia-mimetic agents inhibit proliferation and alter the morphology of human umbilical cord-derived mesenchyma stem cells. BMC Cell Biol 12: 32, 2011.

25. Callens C, Coulon S, Naudin J, Radford-Weiss I, Boissel N, Raffoux E, Wang PH, Agarwal S, Tamouza H, Paubelle E, et al: Targeting iron homeostasis induces cellular differentiation and synergizes with differentiating agents in acute myeloid leukemia J Exp Med 207: 731-750, 2010

26. Stein GS, Lian JB and Owen TA: Relationship of cell growth to the regulation of tissue-specific gene expression during osteoblast differentiation. FASEB J 4: 3111-3123, 1990.

27. Sun J, Zhang D, Zheng Y, Zhao Q, Zheng M, Kovacevic Z and Richardson DR: Targeting the metastasis suppressor, NDRG1, using novel iron chelators: Regulation of stress fiber-mediated tumor cell migration via modulation of the ROCK1/pMLC2 signaling pathway. Mol Pharmacol 83: 454-469, 2013.

28. Chen Z, Zhang D, Yue F, Zheng M, Kovacevic Z and Richardson DR: The iron chelators Dp44mT and DFO inhibit TGF- $\beta$-induced epithelial-mesenchymal transition via up-regulation of N-Myc downstream-regulated gene 1 (NDRG1). J Biol Chem 287: 17016-17028, 2012.

29. Hinz B, Gabbiani G and Chaponnier C: The NH2-terminal peptide of alpha-smooth muscle actin inhibits force generation by the myofibroblast in vitro and in vivo. J Cell Biol 157: 657-663, 2002.

30. Modarressi A, Pietramaggiori G, Godbout C, Vigato E, Pittet B and Hinz B: Hypoxia impairs skin myofibroblast differentiation and function. J Invest Dermatol 130: 2818-2827, 2010.
31. Faulknor RA, Olekson MA, Nativ NI, Ghodbane M, Gray AJ and Berthiaume F: Mesenchymal stromal cells reverse hypoxia-mediated suppression of $\alpha$-smooth muscle actin expression in human dermal fibroblasts. Biochem Biophys Res Commun 458: 8-13, 2015.

32. Jin H, Terai S and Sakaida I: The iron chelator deferoxamine causes activated hepatic stellate cells to become quiescent and to undergo apoptosis. J Gastroenterol 42: 475-484, 2007.

33. Fujii S, Maeda H, Tomokiyo A, Monnouchi S, Hori K, Wada N and Akamine A: Effects of TGF- $\beta 1$ on the proliferation and differentiation of human periodontal ligament cells and a human periodontal ligament stem/progenitor cell line. Cell Tissue Res 342: 233-242, 2010.

34. Zimmerman KA,GrahamLV,Pallero MA and Murphy-UllrichJE: Calreticulin regulates transforming growth factor- $\beta$-stimulated extracellular matrix production. J Biol Chem 288: 14584-14598, 2013.

35. Alizadeh N, Pepper MS, Modarressi A, Alfo K, Schlaudraff K, Montandon D, Gabbiani G, Bochaton-Piallat ML and Pittet B: Persistent ischemia impairs myofibroblast development in wound granulation tissue: A new model of delayed wound healing. Wound Repair Regen 15: 809-816, 2007.

36. McMahon S, Charbonneau M, Grandmont S, Richard DE and Dubois CM: Transforming growth factor betal induces hypoxia-inducible factor-1 stabilization through selective inhibition of PHD2 expression. J Biol Chem 281: 24171-24181, 2006.

37. Nagatomo K, Komaki M, Sekiya I, Sakaguchi Y, Noguchi K, Oda S, Muneta T and Ishikawa I: Stem cell properties of human periodontal ligament cells. J Periodontal Res 41: 303-310, 2006.

38. Murakami Y,Kojima T, Nagasawa T, Kobayashi H and Ishikawa I Novel isolation of alkaline phosphatase-positive subpopulation from periodontal ligament fibroblasts. J Periodontol 74: 780-786, 2003.

39. Wu C, Zhou Y, Lin C, Chang J and Xiao Y: Strontium-containing mesoporous bioactive glass scaffolds with improved osteogenic/cementogenic differentiation of periodontal ligament cells for periodontal tissue engineering. Acta Biomater 8: 3805-3815, 2012.

40. Vimalraj S, Arumugam B, Miranda PJ and Selvamurugan N: Runx2: Structure, function, and phosphorylation in osteoblast differentiation. Int J Biol Macromol 78: 202-208, 2015.

41. Jensen ED, Gopalakrishnan R and Westendorf JJ: Regulation of gene expression in osteoblasts. Biofactors 36: 25-32, 2010.

42. Elanagai R, Veeravarmal V and Nirmal RM: Osteopontin expression in reactive lesions of gingiva. J Appl Oral Sci 23: 26-32, 2015

43. Mathews S, Bhonde R, Gupta PK and Totey S: A novel tripolymer coating demonstrating the synergistic effect of chitosan, collagen type 1 and hyaluronic acid on osteogenic differentiation of human bone marrow derived mesenchymal stem cells. Biochem Biophys Res Commun 414: 270-276, 2011.

44. Zhao GY, Zhao LP, He YF, Li GF, Gao C, Li K and Xu YJ: A comparison of the biological activities of human osteoblast hFOB1.19 between iron excess and iron deficiency. Biol Trace Elem Res 150: 487-495, 2012. 